Hamburg

\title{
JANEZ OREŠNIKS BEITRAG ZUR ERFORSCHUNG DER ISLÄNDISCHEN SPRACHE
}

Die Veröffentlichung eines neuen Buches über das moderne Isländisch ist ohne Zweifel ein bedeutendes Ereignis. Es ist nicht deshalb bedeutend, weil es selten vorkommt, sondern auch deshalb, weil die Erforschung der modernen isländischen Sprache erst in den Anfängen steckt. Es gibt bisher nur eine relativ geringe Anzahl von Arbeiten über die moderne gesprochene Sprache und nur wenige von diesen Arbeiten sind unbefangen, $d . h$. versuchen die Sprache $z u$ beschreiben, wie sie wirklich ist. Meistens stellen diese Arbeiten die Sprache dar, wie sie sein sollte, d. h. normativ, nach einer Norm, die der Forscher sich selbst gesetzt hat oder der sich der Forscher anzupassen versucht. Ein gutes Beispiel für einen derartigen Ansatz sind die Untersuchungen von Björn Guđfinnsson zu Aussprachevarianten in Island (Gữfinnsson 1946, 1947, 1964), die hier stellvertretend zitiert seien, aber die Beispiele ließen sich vermehren. Die Aufgabe eines Forschers ist nicht die Herstellung einer Norm. Er soll auch nicht die Sprache so beschreiben, wie sie nach seiner Meinung zu sein hat. Er soll beobachten, wie die Sprache in der täglichen Situation verwendet wird, und er soll versuchen zu beschreiben, wie diese Verwendung vor sich geht. Seine Daten müssen möglichst objektiv gesammelt sein, und seine Interpretation darf die Objektivität der Daten nicht verletzen.

Es bedarf keines Kommentars, daß dieser Weg sehr schwierig ist. Man ist immer geneigt, die Daten von vornherein so zusammenzutragen, wie sie am besten zu vorgefaßten Meinungen passen. Völlig objektiv, d. h. ganz ungebunden oder unbefangen, kann man wahrscheinlich kaum an ein Forschungsobjekt herangehen, aber der Forscher muß sich darum bemühen, einen möglichst hohen Anteil von Objektivität zu erhalten. Im Falle des modernen Isländischen ist dies ganz besonders schwierig, denn die moderne Sprache wird an dem Maßstab der alten Sprache, welche man Altnordisch zu nennen pflegt, gemessen. Die moderne Sprache wird als Verfall gegenüber der alten Sprache angesehen, und man ist bemüht, z. B. in der Orthographie, sie möglichst nahe dem Ideal der alten Sprache zu betrachten. Deshalb hat kaum jemand den Versuch gewagt, modernes Isländisch so zu beschreiben, wie es wirklich gesprochen wird. Die Zeit war bisher einfach dafür nicht reif, aber das alte Vorurteil, daß in Island ein besonders geringer Abstand zwischen Schriftsprache und gesprochener Sprache vorhanden ist, geringer als sonst irgendwo, lebt weiter und wird häufig in verschiedenen Schriften wiederholt.

Das vorliegende Buch von Janez Orešnik (1985) ist das erste, in dem versucht wird, modernes Isländisch rein synchronisch ohne Bezugnahme auf Gegebenheiten der alten Sprache zu beschreiben. Allein dieser Versuch ist ein Ereignis, aber das Ereignis ist in Grunde genommen größer, denn die zwölf in diesem Buch gesammelten 
Aufsätze stellen nur einen Teil seiner Untersuchungen zum Thema "modernes Isländisch" dar. Man kann die bisher veröffentlichten Arbeiten in drei Gruppen einteilen: (1) Untersuchungen zur Morphologie, (2) Untersuchungen zur Phonologie und (3) Besprechungen von Büchern, die irgendeinen Aspekt des modernen Isländischen zum Gegenstand haben. Das vorliegende Buch enthält eine Auswahl von Arbeiten der beiden ersten Gruppen und dazu kommt auch die Gesamtbibliographie der bis 1983 veröffentlichten Arbeiten zum Thema "modernes Isländisch".

\section{KURZER KOMMENTAR ZU DEN EINZELNEN ARBEITEN}

Im folgenden soll versucht werden, die einzelnen Arbeiten durch stichwortartige Inhaltscharakterisierug kurz zu beschreiben. Anschließend werden wir versuchen, eine Bilanz zu ziehen. Es ist sehr schwierig, ein so gründlich durchdachtes Werk wie das von Janez Orešnik zu charakterisieren. Wenn die Besprechungen mitgezählt werden, handelt es sich um Arbeiten, die über einen Zeitraum von 20 Jahren erschienen sind. Uns ist von vornherein bewußt, daß jeder Versuch einer kurzen Charakterisierung unvollständig bleiben muß. Trotzdem wagen wir diesen Versuch in der Hoffnung, die Aufmerksamkeit auf diese Arbeiten zu lenken, die trotz ihrer Wichtigkeit so wenig diskutiert worden sind, obwohl man gerade das Gegenteil erwartet hätte.

Obwohl die Arbeiten Janez Orešniks in morphologische und phonologische Arbeiten eingeteilt werden können, ist die Grenze zwischen diesen Gruppen keineswegs scharf. Die phonologischen Arbeiten sind in den allermeisten Fällen morphophonemisch, d. h. sie haben zum Gegenstand Erscheinungen, die für die morphologische Gestaltung von Bedeutung sind. Trotzdem haben wir es im Rahmen dieses Überblicks für richtig angesehen, die Teilung in zwei Gruppen beizubehalten. Die Besprechungen $(1965,1975 \mathrm{c}, 1978 \mathrm{c}, 1978 \mathrm{~g}, 1978 \mathrm{~h})$ fügen wir der Liste der Veröffentlichungen nur als bibliographische Angaben ohne weitere Kommentare hinzu. Da die Arbeiten zur Morphologie weniger zahlreich sind, werden wir sie zuerst behandeln.

\section{DIE ARBEITEN ZUR MORPHOLOGIE}

1. Zeitlich gesehen leitet die Arbeit "A philological miscellany on the Icelandic verbs kefja, ljá, cexa, skepja, sýsa" (1969) die Liste der Arbeiten zur Morphologie ein. Sie behandelt einige Kuriosa, nicht existente Verbformen, die durch Abschreibe- oder Druckfehler entstanden sind, und von dort in Wörterbücher und Grammatiken hineingewandert sind, aber in der Tat nie existiert haben.

2. "On some weak preterite subjunctives of otherwise strong verbs in modern Icelandic" (1971). Diese ausführliche Arbeit behandelt einige schwache Subjunktivformen von Verben, die normalerweise starke Subjunktivformen haben, z. B. deyja "sterben" Subj. Prät. dceđi statt der normalen form dcei. Es wird zuerst bewiesen, daß diese Formen wirklich existieren. Bis diese Arbeit geschrieben wurde, hatte 
wohl niemand voll zur Kenntnis genommen, daß die schwachen Subjunktivformen tatsächlich in der modernen Sprache vorhanden sind. Größtenteils sind alle diese Formen wirklich vorkommende Formen der gesprochenen Sprache, obwohl sie nie von der Schulgrammatik als richtig angesehen wurden.

3. Die beiden Arbeiten "Inflection of modern Icelandic nouns, adjectives, and adverbs" (1976b) und "Inflection of modern Icelandic verbs and pronouns" (1978a) enthalten Listen moderner isländischer Substantive, Adjektive, Adverbien, Verben und Pronomina, die nach Stamm und Endungen analysiert werden. Es wird systematisch untersucht, welche phonologische Regeln in jedem einzelnen Fall gelten. Ein neuer Gesichtspunkt ist dabei, daß die Endungen, die in einigen Fällen nicht nur den letzten Segmenten des Stammes zugefügt werden, sondern das letzte Segment des Stammes in einigen Fällen ersetzen können, eine neue Klassifizierung des betreffenden Wortes ermöglichen. Als Beispiel zitieren wir hier: Eine Endung wird zugefügt, wenn sie an das letzte Element des Stammes angehängt wird, z. B. bord "Tisch", Gen. Sing. boro-s. Wenn eine Endung das letzte Segment eines Stammes ersetzt, wird sie als Substitution angesehen, z. B. in stór- "groß" (altisl. stórr), Dat. Sing. Fem. stórri oder annar" "der zweite“", Akk. Sing. Mask. annan (*annar +n) (1976b, pp. 98-99).

Beide Arbeiten enthalten eine überwältigende Fülle von Material, aber vielleicht werden wegen der Materialmenge allgemeine Tendenzen nicht so deutlich hervorgehoben.

4. "Three modern Icelandic morphophonemic notes" (1977d) hat drei voneinander völlig unabhängige morphologische Bemerkungen zum Inhalt. Die erste Bemerkung betrifft die Subjunktivform seigöi (statt der üblichen form segð̇i) von segja "sagen". Nach Meinung von Janez Orešnik wird $e i$ aus den Präsensformen des Verbs abgeleitet (aus segi [seij1] "'(ich) sage"), was eine interessante Analogie aufweist. Die zweite Bemerkung betrifft die Form pú sést "du seiest" statt der üblichen Form pú sért. Die Form mit -st ist eine analogische Formübertragung von den Verben, in denen die zweite Person Sing. mit -st gebildet wird, wie man besonders gut aus der Form pú sérst "du seiest" ersehen kann, die in der gesprochenen Sprache häufig vorkommt. Die Bildung von pú sést "du seiest" ist demanch ganz regelmäBig:

durch Wegfall des $r$.

$$
\text { pú sért }>\text { pú sérst }>\text { pú sést }
$$

Die dritte Bemerkung betrifft die Form skininn "verdorren" (Part. Perf.). Im Dativ Singular wäre die zu erwartende Form *skinnu (Dat. Sing.) in Analogie zu gegnu (Dat. Sing. von gegninn" gehorsam"). Das $n$ der Endung bildet mit dem $n$ des Stammes ein langes [n:], das gekürzt wird, vermutlich durch Analogie mit Nominativ und Akkusativ.

5. "The age and importance of the modern Icelandic word type klifr" (1978b). 
Diese Arbeit enthält eine genaue Liste der auffindbaren Beispiele des isolierten morphologischen Typs klifr. Anhand der Beispiele wird gezeigt, daß die Regel, die ein $-u$ - zwischen einem Konsonanten und einem nachfolgenden $-r$ einschiebt(z. B. im altisl. maðr mod. isl. maður) in der modernen Sprache nicht mehr wirksam ist.

6. Die beiden Arbeiten "On the modern Icelandic clipped imperative" (1980a) und "Um stýfơan bođhátt í íslensku" (1980b) sind der erste Versuch, das Problem des gekappten Imperativs überhaupt zu behandeln. Der isländische Imperativ ist eine sonderbare Erscheinung, da er normalerweise nur mit dem angehängten Pronomen verwendet werden kann. Wenn das Pronomen wegfällt, entsteht der sogenannte gekappte oder apokopierte Imperativ, der für gesprochenes modernes Isländisch typisch ist, z. B.:

$$
\begin{aligned}
& \text { haltu "halt" }>\text { halt }>\text { halt } p u ́ \\
& \text { taktu "nimm" }>\text { takt }>\text { takt pú }
\end{aligned}
$$

Der Autor hat eine Reihe alter Beispiele aus der Literatur zusammengetragen. Mit zusätzlichen Beispielen aus der modernen Sprache versucht er die Wege zu rekonstruieren, die zur Bildung der neuen Form geführt haben. Am wahrscheinlichsten ist es, daß die Endung $-u$ nicht mehr als richtiges Pronomen empfunden wurde. Die Verben, die $-d$ oder $-t$ in ihrem Stamm haben wie henda "werfen", halda "halten" und velta "drehen", wurden als Analogiebasis genommen, um für andere Verben einen neuen Stamm zu bilden, der auf $-t,-\delta$ oder $-d$ endete (z. B. takt [statt tak] "nimm", gefठ [statt gef] "gib", semd [statt sem] "'verhandele"), an der wieder das Pronomen pú angehängt wurde. So entstehen im Isländischen die gekappten Imperative, die in der gesprochenen Sprache sehr charakteristisch sind, die aber von den Grammatikern niemals als Formen anerkannt worden sind. Sie haben deshalb kaum in Grammatiken oder in Wörterbüchern Eingang gefunden.

Orešniks Arbeiten zum gekappten Imperativ haben ein absolutes Neuland in der Erforschung des Isländischen geöffnet. Zum ersten Mal war im Isländischen ein Gebiet für die Forschung erschlossen worden, in dem in der gesprochenen Sprache etwas nachgewiesen werden konnte, was man bisher kaum zu Kenntnis genommen oder nur vage geahnt hatte.

7. Es lag nun nahe, dieses Gebiet weiter zu erforschen. In umfangreichen systematischen Feldforschungen konnte Orešnik in zwei bisher veröffentlichten Arbeiten "On some Icelandic irregular imperative singular forms" (1981) und "Icelandic imperative singular: Some innovations" (1984) nachweisen, daß die Wege, die zur Bildung der neuen Form führen, nicht immer so regelmäßig sind, wie es den ersten Arbeiten erschien. Es gibt z. B. die Möglichkeit, die apokopierte Form aus dem Präteritum zu bilden, z. B. attu "sporne an" von etja "anspornen" (Prät. atti) oder auf noch andere Art, z. B. durch Mischen oder Kreuzen der Präsens- oder Präteritalstämme. Diese Wege werden systematisch untersucht und in einigen Fällen sind verblüffende Analogien entdeckt worden. 
Orešniks Arbeiten zum gekappten oder apokopierten Imperativ haben endgültig den Beweis gebracht, daß die Kluft zwischen gesprochenem und geschriebenem Isländisch viel größer ist als man allgemein annimmt. Aber die wirklich gesprochene Sprache zu untersuchen, gehört zu den schwierigsten Aufgaben linguistischer Forschung. Es ist deshalb zu erwarten, daß noch Zeit vergehen wird, bis über diese Frage einige Klarheit gewonnen werden kann.

\section{DIE ARBEITEN ZUR PHONOLOGIE}

Arbeiten zu Phonologie sind naturgemäß weniger spektakulär als die Arbeiten zur Morphologie, die dem Sprecher normalerweise näher steht, da es sich in der Morphologie um bedeutungstragende Einheiten handelt. Die Phonologie behandelt Einheiten und Prozesse unterhalb der semantischen Ebene und ist deshalb dem Sprecher nicht so unmittelbar bewußt.

Orešniks Arbeiten zur isländischen Phonologie sind nicht weniger sorgfältig als seine Arbeiten zur Morphologie. Im folgenden soll versucht werden, einen Gesamtüberblick über diese Arbeiten zu geben, um sie anschließend zu würdigen. Dabei scheint es am besten zu sein, chronologisch vorzugehen, um die Entwicklung deutlicher hervorzuheben:

1. In der chronologisch gesehen ersten Arbeit "On the phonological boundary between constituents of modern Icelandic compound words" (1971b) wird gezeigt, daß Ansetzen einer Wortgrenze in zusammengesetzten Wörtern eine große Anzahl phonologischer Erscheinungen erklären kann, ohne daß irgendwelche zusätzliche Regeln notwendig sind, um die phonologische Form zu beschreiben. Als Beispiel könnten wir hier zitieren, daß zwischen $r$ und $n$ in stórnetla "urtica dioeca" (Pflanzenname) kein - $d$ - eingeschoben wird, obwohl normalerweise in nicht zusammengesetzten Wörtern ein $-d$ - zwischen $r$ und $n, z$. B. in varla [vardola, vadila] "kaum " und hérna [çerḍna, çeḍna] "hier" , eingeschoben wird. Die Annahme einer phonologischen Wortgrenze vereinfacht in vielen Fällen die Beschreibung. Das bedeutet jedoch nicht, daß eine Wortgrenze immer postuliert werden muß.

2. Die Arbeiten "On the epenthesis rule in modern Icelandic" (1972a) und "The modern Icelandic epenthesis rule revisited" (1981d) behandeln beide das gleiche Thema, die Epenthese-Regel, welche ein - $u$ - zwischen einem Konsonanten und einem nachfolgenden $r$ einsetzt. Im ersten Artikel wird argumentiert, daß die Regel im modernen Isländischen noch wirksam ist. Das epenthetische $-u$ - (phonetisch [Y]) bewirkt im Gegensatz zum ursprünglichen $u$ keinen u-Umlaut (vgl. maður "Mann“" vs. fögrum "schönem", Dat. Sing. von fagur "schön"). Aufgrund der Alternanz von Formen wie lifur "'Leber" vs. lifrin"'die Leber" wird argumentiert, daß die Regel im modernen Isländischen noch wirksam ist. Im zweiten Artikel wird diese Schlußfolgerung zurückgezogen. Es werden neue Argumente dafür gebracht, daß die Regel seit dem 17. Jahrhundert nicht mehr wirksam ist. Danach besteht keine 
Notwendigkeit für eine besondere Regel für Enkliktika, sondern es scheint besser zu sein, zwischen Artikel und Wortstamm eine Morphemgrenze anzusetzen. Die neuen Daten, die zu dieser Schlußfolgerung führten, wurden bereits unter dem Stichwort "'Morphologie" besprochen (Orešnik 1978b).

Die Arbeit "Morphophonemic notes on the modern Icelandic imperative singular" (1972b) behandelt morphophonemische Besonderheiten der kurzen Imperativformen in der 2. P. Sing. Diese Imperativform ist mit dem Verbstamm identisch. Es wird eine Apokoperegel postuliert, und für die 1. P. Pl. Konj. Prät. wird die Palatalisierungsregel ausführlich erläutert. Es werden ebenfalls Argumente für eine Regelordnung gebracht.

4. In "Four modern Icelandic devoicing rules" (1972c) werden die phonologischen Regeln, die die Entsonorisierung des Auslautkonsonanten eines Wortstammes vor bestimmten Endungen bewirken, ausführlich erläutert: z. B. die Regel, die $l, m$ und $[\gamma]$ vor $t$ und $s$ entsonorisiert: sval-t [svalt] "kühl", skamm-t [skamt] "kurz", dag-s [daxs] "Tages" (gen. Sing. von dagur"'Tag" ).

5. "Old Icelandic consonant lengthening rule and modern Icelandic infixation of /d/“" (1973). Das Altisländische hatte in seiner morphologischen Komponente eine Verlängerungsregel, z. B. Nom. Sing. stóll [sto:1:] "Stuhl““. Diese Regel verlängerte $-l,-n,-s$ unter bestimmten morphologischen Bedingungen. Die Fälle werden ausführlich erläutert. Die Regel verschwand vermutlich im 16. Jh. und wurde durch ein neues morphologisches Mittel zur Kennzeichnung der Endungen ersetzt. Dies ist das Infix /d/, das in stóll [stoudl] "Stuhl" und einn [eidn] "ein" die Flexionsform entweder allein oder mit anderen Endungen markieren kann.

6. 'Moderne islandsk generativ fonologi - et eksempel" (1975a) diskutiert die phonologische Implikation, die sich aus der Aufnahme vom Auslauts-r ergibt (z. B. in klifr "(das) Klettern"), das durch das Wirken der Epentheseregel verschwunden war.

7. "The modern Icelandic u-umlaut rule" (1975b). Dies ist eine der originellsten Arbeiten, die Janez Orešnik zum Isländischen geschrieben hat. Sie beschreibt die Wirkung des u-Umlautes in modernen Isländischen, welcher ein [a] zu [ö] oder [Y] vor einem ursprünglichen $-u$ wandelt. Diese Regel ist in der modernen Sprache noch so wirksam, daß sie sogar neue Lehnwörter und Eigennamen erfaßt. Dieser Tatbestand ist besonders interessant, da in allen anderen germanischen Sprachen der Umlaut als historisch abgeschlossener Prozeß zu betrachten ist. Orešnik unterscheidet zwischen phonologischem und morphologischem Umlaut. Zahlreiche Sonderfälle werden diskutiert und die u-Umlaut-Regel wird vorgeschlagen und formalisiert.

In "'Modern Icelandic u-Umlaut from the descriptive point of view" (1977c) wird das gleiche Problem behandelt. Dort wird gezeigt, daß es für Beschreibungs- 
zwecke nützlich sein kann, zwischen ein- und mehrsilbigen Wörtern zu unterscheiden. Gewisse Wörter können die beiden in der modernen Sprache vorkommenden Umlautvarianten haben. Diese Fälle werden ausführlich diskutiert.

8. In der Arbeit 'Über die Lautalternationen im neuisländischen Typus veggur" (1976a) wird die Alternanz von palatalen und velaren Konsonanten in verschiedenen Flexionsformen des Typus veggur" "Wand" diskutiert. Es wird argumentiert, daß eine phonologische Lösung unzureichend ist und daß eine morphologische Lösung vorzuziehen sei.

9. In der Arbeit "On the modern Icelandic palatalisation rule" (1977a) wird die interessante Tatsache diskutiert, daß die Palatalisierungsregel, die $g$ und $k$ vor vorderen Vokalen zu palatalen Konsonanten verwandelt, nur vor [I] wirksam geblieben ist. Zahlreiche Fremdwörter wie z. B. Genf, gaei 'Kerl' zeigen, daß die Regel in der modernen Sprache ihren Wirkungsbereich stark eingeschränkt hat.

10. Janez Orešnik und ich selbst haben zusammen die Arbeit "'Quantity in modern Icelandic" (1977b) geschrieben. Wir zeigen, daß die Stellung der Quantität im Nord- und Südisländischen unterschiedlich ist. Die südisländische Quantität wird durch morphologische und phonologische Regeln bestimmt; die nordisländische Quantität aber ausschließlich đurch phonologische Regeln. Die Resultate sind für diejenigen überraschend, die annehmen, daß Isländisch mit der orthographischen Form fast identisch ist. Es deshalb wichtig zu betonen, daß es sich hier nicht um eine historische Betrachtungsweise handelt, sondern um eine rein synchronische Beschreibung. Daß es Quantitätsunterschiede zwischen Nord- und Südisländisch gibt, ist in der Tat völlig neu, aber nicht unbedingt überraschend. Solche Entwicklungen in unterschiedlichen Stadien sind aus den übrigen skandinavischen Sprachgebieten bekannt.

11. Die Arbeit "On the modern Icelandic i-umlaut rule" (1978e) bringt Argumente dafür, daß im modernen Isländischen keine i-Umlaut-Regel mehr wirksam ist. Die i-Umlaut-Erscheinungen gehören in der modernen Sprachen zu den Ablauterscheinungen. Die gegenteiligen Meinungen von Stephen R. Anderson und Sigrid Valfells werden ausführlich diskutiert und abgelehnt.

12. In der Arbeit "Modern Icelandic preaspiration from the phonological point of view" (1978f) werden ausführlich die phonetisch/phonologischen Bedingungen der Präaspiration im Isländischen diskutiert. Teilweise ist die Präaspiration lexikalisiert (z. B. boeklingur [baihklimkYr] "Pamphlet"), aber teilweise morphologisch (kaplar [khahplar] 'Kabel" PI. von kapall "Kabel"', Nom. Sing.). Die Implikationen, die sich aus diesem Tatbestand ergeben, werden diskutiert. Als Anhang zum Artikel wird eine neue und genauere Formulierung der Quantitätsregeln im modernen Isländischen vorgeschlagen (vgl. 1977b). 
13. "'On the pronuncation of modern Icelandic rövl(a) and slafneskur" (1979). Die Aussprache dieser Lehnwörter mit den Lautverbindungen [vl] und [vn] entspricht nicht den isländischen Gepflogenheiten, da man [bl] und [bon] erwarten würde (wie z. B. in efni [ebnI] "Stoff" , efla [ebla] "fördern"). Diesen Widerspruch erklärt Janez Orešnik aufgrund von. Kiparskys Universale, nach der phonologische Regeln nur auf abgeleitete Formen angewendet werden können. Aufgrund des Beispiels slafneskur muß diese Universale jedoch ein wenig anders formuliert werden. Eine Neuformulierung wird ebenfalls vorgeschlagen.

14. "On the dental accretion in certain 2 nd person sing. verbal forms of Icelandic, Faroese and the old West Germanic languages" (1980c). Die Endung $-t$ in der 2. P. Sg. des Verbs ist häufig als Entlehnung und Analogie des Pronomes pú, $d u$ erklärt worden. Es werden typologische Parallelen zu den westgermanischen Sprachen gezogen. Die Entwicklung des isländischen Imperativs bietet hier ganz neue Wege zur Erklärung, die in dem Artikel genauer untersucht werden.

15. "On the lack of palatalization before -end-in the plural of Icelandic nominalised present participales such as leikandi" (1980d). In dem isländischen Partizip Präsens wird ein velarer Konsonant vor einem $-e$-nicht palatalisiert, es heißt also leikendur [lei:kend̦Yr] 'Schauspieler" (PI.) und nicht *[lei:cend̆Yr]. Janez Orešnik erklärt diese Tatsache aufgrund der Universale Kiparskys, nach der phonologische Regeln nur auf abgeleitete Formen angewendet werden dürfen. Orešnik schlägt vor, daß diejenige segmentale Umgebung einer Morphemgrenze nicht als abgeleitet gelten soll, die ausschließlich durch die Wortbildung motiviert ist.

16. "An old Icelandic dialect feature: iæ for ææ" (1982). Diese Arbeit untersucht die graphischen Varianten vicenn, vienn statt voenn "lieb", wie sie hauptsächlich in Manuskripten und Dokumenten aus dem 14. Jh. vorkommen. Die meisten dieser Dokumente stammen aus Nordisland, aber andere aus dem westlichen Teil des Landes aus der Gegend um Breiđafjörđur. Es wird gezeigt, daß es sich hier um die Reste einer Lautenwicklung handelt, die nicht verallgemeinert wurde. Nachdem die philologische Seite des Problems erschöpfend behandelt worden ist, folgen historisch-phonologische Betrachtungen zum Thema.

\section{VERSUCH EINER BILANZ}

Wenn wir nun versuchen, eine Bilanz der Arbeiten Janez Orešniks zu ziehen, die er seit 1965 - also über einen Zeitraum von 20 Jahren - der Erforschung des Isländischen gewidmet hat, stehen wir vor keiner leichten Aufgabe. In diesem Zeitraum sind 34 Arbeiten erschienen. Sie behandeln überwiegend die moderne isländische Sprache, obwohl einige auch historischen Fragestellungen nachgehen.

Die Erforschung des modernen Isländischen ist bisher stark vernachlässigt worden, weil die Tätigkeit der Forscher sich vorwiegend dem Altnordischen zugewandt hat. Es ist noch nicht richtig ins Bewußtsein der fachlichen Welt eingedrungen, wel- 
che enorm großen Unterschiede das moderne Isländisch und das Altnordische trennen. Bekannt ist selbstverständlich, daß das Lautsystem des modernen Isländischen völlig anders gestaltet ist. Die Neugestaltung der Quantitätsverhältnisse um 1540 bildet traditionell die Trennlinie zwischen altem und modernem Isländisch. Obwohl die Morphologie sich relativ wenig geändert hat, sind die lautlichen Unterschiede derart groß, daß eine Verständigung mit einem Sprecher des Altnordischen heute nicht möglich wäre, angenommen ein solcher Fall könnte eintreten. Im diesem Sinne ist die Lage des Isländischen ähnlich der des heutigen Griechisch im Verhältnis zum Griechischen des klassischen Altertums.

Die Arbeiten Janez Orešniks sind sozusagen die ersten, die der Fachwelt vor Augen geführt haben, wie groß die Unterschiede sind, die das Altnordische und das moderne Isländische trennen. Sie haben gezeigt, wie vielschichtig und wenig einheitlich das moderne Isländisch in seiner gesprochenen Form ist, selbst in der Morphologie, wo man doch den Eindruck hat, daß die Einheitlichkeit besonders groß ist. Es wird noch einige Zeit vergehen, bis diese Einsicht sich in der Fachwelt durchgesetzt hat, aber ein positives Zeichen ist immerhin, daß man beginnt, die gesprochene Sprache zu untersuchen. Der Mythos, daß der Unterschied zwischen gesprochenem und geschriebenem Isländisch besonders gering sei, ist auf keinen Fall aufrechtzuerhalten. Hier haben die Arbeiten Orešniks ein neues Gebiet erschlossen, in dem es noch viel zu erforschen gibt.

Eine weitere Eigenschaft Orešniks, die in vielen Arbeiten zum Ausdruck kommt, ist seine ständige Bereitschaft, eigene Meinungen zu modifizieren, und seine Fähigkeit neue Gesichtspunkte zu integrieren. Dies ist besonders deutlich in den Arbeiten zur Morphologie, vielleicht etwas weniger in den phonologischen Arbeiten, da es sich in der Phonologie um Bereiche handelt, die in ihrem Wesen etwas mechanischer gestaltet sind. Die Charakteristik der gesprochenen Sprache ist ihr Dynamismus. Eine flexible Haltung, wie sie Janez Orešnik immer vertreten hat, ist sozusagen die Bedingung dafür, daß man ein auf den ersten Blick heterogenes Material überhaupt interpretieren kann. Besonders trifft das für das Material zu, das Orešnik in seinen mit großer Geduld durchgeführten Feldforschungen zusammengetragen hat.

Fassen wir unsere Bilanz kurz zusammen: Das neue Buch Janez Orešniks, das etwa ein Drittel seiner Arbeiten zum Thema modernes Isländisch enthält, ist eine äuBerst originelle Aufsatzsammlung. Es wird sicher in der Fachwelt beachtet werden, nicht zuletzt wegen der neuen Perspektiven, die es für die Erforschung des modernen Isländischen eröffnet. 


\section{BIBLIOGRAPHIE DER ARBEITEN JANEZ OREŠNIKS ZUM ISLÄNDISCHEN}

1. (1985) Studies in the Phonology and Morphology of Modern Icelandic. A selection of essays. Helmut Buske Verlag, Hamburg 1985. $228 \mathrm{~S}$.

\section{Arbeiten zur Morphologie}

2. (1969) "'A philological miscellany on the Icelandic verbs kefja, ljá, cexa, skepja, sýsa." Linguistica 9, 1969, pp. 49-52.

3. (1971) "On some weak preterite subjunctives of otherwise strong verbs in modern Icelandic". Arkiv för Nordisk Filologi 86, 1971, pp.139-178.

4. (1976b) "Inflection of modern Icelandic nouns, adjectives, and adverbs". Linguistica 16, 1976, pp. 97-118.

5. (1977d) "'Three modern .Icelandic morphophonemic notes". Sjötíu ritgerđir helgað̃ar Jakobi Benediktssyni 20. júlí 1977, pp. 621-626. Stofnun Árna Magnússonar á Íslandi, Reykjavík 1977.

6. (1978a) "'Inflection of modern Icelandic verbs and pronouns". Linguistica 17 , 1978 , pp. $91-122$.

7. (1978b) "The age and importance of the modern Icelandic word type klifr" . The Nordic Languages and Modern Linguistics 3, pp. 468-471 (ed. by John Weinstock). The University of Texas at Austin, Austin 1978.

8. (1980a) "'On the modern Icelandic clipped imperative". The Nordic Languages and Modern Linguistics [4], pp. 305-314 (ed. by Even Hovdhaugen). Universitetsforlaget, Oslo 1980.

9. (1980b) "Um stýfđan bođhátt í íslensku“. Skíma 3/3, 1980, pp. 7-9.

10. (1981) "On some Icelandic irregular imperative singular forms". Afmoeliskveđja til Halldórs Halldórssonar 13. júlí 1981, pp. 211-218. Íslenska Málfræđifélagiđ, Reykjavík 1981.

11. (1984) 'Icelandic imperative singular: Some innovations". The Nordic Languages and Modern Linguistics 5, pp. 337-341 (ed. by Kristjan Ringgaard og Viggo Sørensen). Nordisk Institut, Aarhus 1984.

\section{Arbeiten zur Phonologie}

12. (1971b) "On the phonological boundary between constituents of modern Icelandic compound words". Linguistica 11, 1971, pp. 51-59.

13. (1972a) "On the epenthesis rule in modern Icelandic". Arkiv för Nordisk Filologi 87, 1972, pp. 1-32.

14. (1972b) "Morphophonemic notes on the modern Icelandic imperative singular'. Studies for Einar Haugen Presented by Friends and Colleagues, pp. 450-459 (ed. by E. S. Firchow et al.). Mouton, The Hague 1972.

15. (1972c) "'Four modern Icelandic devoicing rules". Linguistica 12, 1972, pp. $137-156$. 
16. (1973) "Old Icelandic consonant lengthening rule and modern Icelandic infixation of $/ d /$. Linguistica 13,1973 , pp. 229-261.

17. (1975a) "Moderne islandsk generativ fonologi - et eksempel". Selskab for nordisk filologi. Arsberetning for 1971-73. 1975, pp. 32-33.

18. (1975b) "The modern Icelandic u-umlaut rule". The Nordic Languages and Modern Linguistics 2, pp. 621-630 (ed. by Karl-Hampus Dahlstedt). Almqvist \& Wiksell, Stockholm 1975.

19. (1976a) 'Über die Lautalternationen im neuisländischen Typus veggur". Skandinavistik 6, 1976, pp. 110-116.

20. (1977a) "On the modern Icelandic palatalisation rule". Dialectology and Sociolinguistics. Essays in honor of Karl-Hampus Dahlstedt 19. April 1977, pp. 137-145 (ed. by C.-C. Elert et al.) Acta Universitatis Umensis, Umeå 1977.

21. (1977b) "Quantity in modern Icelandic". Arkiv för Nordisk Filologi 92, 1977, 155-171 (with Magnús Pétursson as co-author).

22. (1977c) "'Modern Icelandic u-umlaut from the descriptive point of view“. Gripla 2,1977 , pp. 151-182.

23. (1978d) "'The modern Icelandic epenthesis rule revisited". Arkiv för Nordisk Filologi 93, 1978, pp. 166-173.

24. (1978e) "On the Icelandic i-umlaut rule". General Linguistics 18, pp. $181-200$.

25. (1978f) "'Modern Icelandic preaspiration from the phonological point of view" “. Linguistica 18,1978 , pp. 141-165.

26. (1979) "'On the pronunciation of modern Icelandic rövl(a) and slafneskur". Íslenskt mál og almen málfræđi 1,1979 , pp. 225-232.

27. (1980c) "On the dental accretion of certain 2 nd person sg. verbal forms of Icelandic, Faroese, and the old West Germanic languages". Íslenskt mál og almenn málfræòi 2, 195-211.

28. (1980d) "'On the lack of palatalisation before -end-in the plural of Icelandic nominalised present participles such as leikandi“". Linguistica 20, 1980, pp. $2 \dot{4} 5-259$.

29. (1982) "An Old Icelandic dialect feature: iæ for æ’. Gripla 5, 1982, pp. $183-196$.

\section{Besprechungen}

30. (1965) Review of, Sravnitel'naja grammatika germanskix jazykov, vol. 4. Linguistica 7, 1965, pp. 169-175.

31. (1975c) Review of, Magnús Pétursson, Les articulations de l'islandais à la lumière de la radiocinématographie. Paris: Librairie C. Klincksieck 1974. General Linguistics 15, 1975, pp. 120-127.

32. (1978c) Review of, Magnús Pétursson, Drög að hljóðkerfisfraæđi. Reykjavík: Iđunn 1978. Nordic Linguistic Bulletin 2.3, 1978, pp. 22-29.

33. (1978g) Review of, Magnús Pétursson, Isländisch, Hamburg, Helmut Buske Verlag 1978. Linguistica 18,1978 , pp. 261-264. 
34. (1978h) Review of, Einar Haugen, The Scandinavian Languages. An Introduction to their History. Cambridge, Mass.: Harvard University Press; London: Faber and Faber 1976. General Linguistics 18, 1978, pp. 37-47.

\section{Andere zitierte Arbeiten}

Guđfinnsson, Björn: Mállýzkur I. Ísafoldarprentsmiơja H. F. Reykjavík 1974. Zweite Auflage unter dem gleichen Titel als Smárit Kennaraháskóla Íslands og Iđunnar 7. Iđunn, Reykjavík 1981.

Gư̛finnsson, Björn: Mállýzkur II. Ólafur M. Ólafsson og Óskar Ó. Halldórsson unnu úr gögnum höfundar og bjuggu til prentunar. Studia Islandica 23. Heimspekideild Háskóla Íslands og Bókaútgáfa Menningarsjóòs, Reykjavík 1964.

Povzetek

\section{PRISPEVEK JANEZA OREŠNIKA K RAZISKAVAM ISLANDŠČINE}

Cllanek je bil napisan ob izidu nekaterih Orešnikovih islandoloških člankov pod naslovom Studies in the Phonology and Morphology of Modern Icelandic (Hamburg 1985) in obravnava v začetku mesto te knjige $v$ znanosti o islandskem jeziku. $V$ nadaljevanju prikazuje članek dela o islandski fonologiji in oblikoslovju, ki jih je J. Orešnik objavil pred izidom omenjene knjige (ne glede na to, ali so v knjigi ponatisnjena ali ne). V celoti gre za 18 bibliografskih enot pretežno s področja fonologije in za 10 enot pretežno $s$ področja oblikoslovja. Prikazana je vsebina vsake enote, ponekod so dodane drobne kritične opazke in ali namigi na stanje obravnavane problematike pred izidom prikazanega dela. Najugodneje ocenjuje pisec Orešnikova dela o islandskem velelniku. Na koncu članka najde bralec poskus celostne ocene Orešnikovega prispevka $\mathrm{k}$ raziskavam islandščine in bibliografske podatke o njegovih islandoloških delih, objavljenih pred 1985 (vštevši podatke o 5 ocenah/prikazih, ki sicer v članku niso posebej obravnavani). 